\title{
N90-10389:
}

\section{STRESS IN DILUTE SUSPENSIONS}

\author{
Stephen L. Passman*
}

\begin{abstract}
Generally, two types of theory are used to describe the field equations for suspensions. The so-called "postulated" equations are based on the kinetic theory of mixtures, which logically ought to give reasonable equations for solutions. The basis for the use of such theory for suspensions is tenuous, though it at least gives a logical path for mathematical arguments. It has the disadvantage that it leads to a system of equations which is underdetermined, in a sense that can be made precise. On the other hand, the so-called "averaging" theory starts with a determined system, but the very process of averaging renders the resulting system underdetermined. I suggest yet a third type of theory. Here, the kinetic theory of gases is used to motivate continuum equations for the suspended particles. This entails an interpretation of the stress in the particles that is different from the usual one. Classical theory is used to describe the motion of the suspending medium. The result is a determined system for a dilute suspension. Extension of the theory to more concentrated systems is discussed.
\end{abstract}

1. Introduction. In theories of multiphase flows, it is natural to postulate or to derive equations of balance similar to those occurring in the theory of dilute mixtures of gases $[1,2]$. The usual process of doing so, along with reasonable assumptions for the constitutive properties of the materials composing the flow, always leads to a system with more unknowns than equations. Though there is no definitive reason that this is a bad situation, intuition abetted with proved theorems for special types of systems indicates that the normal desirable situation is the same number of equations as unknowns. The resulting quandary for multiphase flows is known as the "closure problem", and methods for "solving" or "closing" it, that is, finding "sufficient" additional equations, has been the focus of considerable research in multiphase flows. Here, we try to shed some light on such problems. Essential to doing so is stating the problems unequivocally. In order to do that, we choose a special but interesting physical situation, then give typical equations of balance and constitutive equations for that physical situation, according to a continuum theory and an averaging theory. The closure problem occurs in both types of theories, though its form is different. However, it is possible to formulate theories in which the closure problem does not occur, and therefore need not be solved. A physical basis for such a system is presented, and a putative set of field equations is suggested.

\section{Determined, Underdetermined, and Overdetermined Systems of Equa-} tions. Assume we have a system of equations of the form

$$
f_{i}\left(y_{j}, D_{k} y_{j}\right)=0
$$

with $i=1, \ldots, n ; j=1, \ldots, m$; and $k=1, \ldots, p$. The $f_{i}$ are $n$ functions of the $m$ variables $y_{j}$ and their derivatives up to order $p$. The system is called determined if $n=m$,

*Pittsburgh Energy Technology Center, Pittsburgh PA 15236, on temporary assignment from Sandia National Laboratories, Albuquerque NM 87185. 
overdetermined if $n>m$, and underdetermined if $n<m$. By our definition, all systems of equations are of one of these three types. Ideally, of course, it would be convenient if determined systems always had solutions and they were unique, if overdetermined systems never had solutions, and if underdetermined systems always had families of solutions. That this is not the case can be shown by examples. To begin, consider the underdetermined system

$$
x_{1}^{2}+x_{2}^{2}=0
$$

Naturally, specifying a system of equations is meaningless without specifying their domain, but since this paper is informal, I follow the convention of doing so tacitly, that is, all functions are mappings of all real variables for which they can be defined reasonably into a range defined by the function. Here, the underdetermined algebraic system (1) has the single unique solution

$$
x_{1}=0, \quad x_{2}=0 .
$$

Now consider the determined system

$$
\begin{aligned}
x_{1}+x_{2} & =1, \\
2 x_{1}+2 x_{2} & =2 .
\end{aligned}
$$

This system does not have a unique solution, rather it has an infinite one-parameter family of solutions. Finally, consider the overdetermined system

$$
\begin{aligned}
x_{1}+x_{2} & =1 \\
2 x_{1}+2 x_{2} & =2 \\
2 x_{1}+4 x_{2} & =4 .
\end{aligned}
$$

This system has a unique solution. All of the examples cited involve algebraic equations, not differential equations, but of course examples of the same type can be constructed with differential systems.

It is easy to object to the arguments above because the systems cited are "special" or "pathological". Indeed, I agree with that type of objection, and in a sense that is just the point of this discussion, for to make such arguments, one must use very special properties of the systems. Furthermore, for each system, it would have been possible to rearrange the system using simple manipulations, obtaining very complicated new systems with exactly the same properties. Proofs of existence or non-existence, uniqueness or non-uniqueness, then would be much more elaborate exercises, perhaps depending on sophisticated mathematics or luck for ultimate outcome.

A different line of argument is possible. Most often, the equations governing multiphase flow are systems of partial differential equations, so complicated that they are not 
easily amenable to existence or uniqueness theorems. Such equations may be relevant to important problems in technology, so important that they must be solved, in no matter in how vague a sense, immediately. More often than not, that means the use of large computer codes. Despite the above examples, we find that an intrinsic part of building such codes is the desire of the numerical analyst for determined systems, that is, systems determined in exactly the sense defined here. The idea that the equations obtained by specialists in multiphase flow are "independent" is supported in some vague sense by the fact that they have different physical meanings. For example, some are balance equations for the constituents, some are constitutive equations, and some are constraints.

3. Continuum Theories of Multiphase Flows. Here, we consider a standard type of theory for multiphase flows, as derived from continuum considerations. ${ }^{1}$ Intrinsic to such considerations is the assertion that each constituent fills all of a region of space. This is the basic assumption of theories of interpenetrating continua or "solutions" [4]. The theory then is made to model a multiphase medium by the inclusion of volume fractions $\phi_{a}$ as basic variables. A typical set of field equations for such a continuum having $n$ constituents is

$$
\begin{gathered}
\Sigma_{a=1}^{n} \phi_{a}=1 \\
\grave{\phi}_{a}+\phi_{a} \operatorname{div} \mathbf{v}_{a}=0 \\
\rho_{a} \grave{\mathbf{v}}_{a}=\rho_{a} \mathbf{b}_{a}+\mathbf{m}_{a}+\operatorname{div} \mathbf{T}_{a} \\
\sum_{a=1}^{n} \mathbf{m}_{a}=0 \\
\left\{\mathbf{T}_{a}, \mathbf{m}_{a}\right\}=g\left(\mathbf{v}_{b}, \phi_{b}, \text { and their derivatives }\right) .
\end{gathered}
$$

Here for simplicity we consider only pure mechanics, and the symbols have the obvious meanings. The first equation expresses the fact that the material is saturated; the second and third are balances of mass and momentum for each of the constituents. Each constituent is assumed to be incompressible, and the $p_{a}$ are the reactions to those constraints. The fourth equation is conservation of mass for the mixture, and the last equations express constitutive properties of the constituents, in particular the dependence of the stress on the deformation rate and other properties for each constituent, and appropriate expressions for interactions of the two materials. We note that these last expressions can be somewhat problematical, and in fact debate about their forms has generated a considerable literature. They are not discussed here. Rather, we assume they are known for applications of particular interest. ${ }^{2}$ Our intuitive feeling from considering the physics of this situation is that such a system of equations is "complete", but in fact that is not the case. Here and henceforth, for the purpose of counting equations, we assume the multiphase flow consists of two constituents. Though such is not always the case, for the purpose of our arguments here, that case is general. The result is a system of 9 equations in the 10 unknowns

\footnotetext{
${ }^{1}$ Discussion of the basis of such theories, as well as references to the standard works, are given in [3].

${ }^{2}$ See [5] and [6] for a discussion of these equations.
} 
$\left\{p_{a}, \phi_{a}, \mathbf{v}_{a}\right\}$, that is, the system is underdetermined. The usual physical motivation for this apparent quandary is plausible: Though Equations (4) express the exchange of momentum between constituents, that is not the only way the constituents interact, for in addition, there should be a force balance between the constituents. The most primitive visualization of this is sufficient for arguments here. That is, the solid phase is considered to consist of spherical particles of one size, surrounded by the fluid phase. Then a radial force balance on a single particle gives

$$
p_{s}=p_{f}
$$

This obvious and elegant closure argument gives a system of equations which is notoriously ill-behaved $[7,8]$, so much so that it must be rejected. More sophisticated arguments are possible, and they sometimes appear to suffice to render the system of equations thus obtained to be at least well-behaved enough to be handled by standard computational techniques. Usually, the arguments adduced are generalizations of those leading to Equation (5) in that they consider a particle in a flow field of a known type at infinity, then use techniques of hydrodynamics to solve or partially solve for the flow field around the spherical particle. Surface tension may be considered also. Of course the resulting pressure on the particle is a function of position on its surface relative to the flow field at infinity, so some sort of integration is required. The result is

$$
p_{s}=p_{f}+f(\Pi, \sigma),
$$

where $\Pi$ denotes properties of the flow field, and $\sigma$ denotes properties sufficient to characterize surface tension. I note that since $f$ depends upon the flow field at infinity, adducing it as a constitutive relation valid for all flows has the potential for leading to inaccurate results. ${ }^{3}$

In addition to the mathematical argument against using Equation (5) as a closure relation, there is a physical argument against it, which also is inherited by Equation (6). In doing the arguments leading to these equations, the assumption is that $p_{s}$ and $p_{f}$ are pressures "in" the respective materials, and that it is appropriate to write an expression for one in terms of the other. The need for the closure relation comes from arguments about the system (4). In this system, the pressures are derived as reactions to constraints. Therefore [10], they are dependent variables of the system of equations, totally independent of one another. In treating the complete system of equations and boundary conditions. ${ }^{4}$ the quantities $p_{s}$ and $p_{f}$ thus cannot be related a priori. Another way to see this is that in fact Equations (4) are field equations for the whole continuum, while the closure relation

\footnotetext{
${ }^{3} \mathrm{~A}$ similar difficulty arises in rheology, where it is commonly known [9] that no constitutive equation giving an accurate representation of the physics of shearing flows also represents stretching flows adequately.

${ }^{4}$ Boundary conditions in themselves constitute a difficult problem for multiphase flows. They are not discussed in this paper.
} 
(6) is not. Rather, it is derived from a "micromechanical" argument, then scaled up in a way the nature of which never is made clear, so that the symbols $p_{s}$ and $p_{f}$ in Equations (6) are assumed to have the same meaning as the same symbols in the closure relation, without proof or explanation. ${ }^{5}$

4. Averaging Theory. The basic ideas behind averaging theories are diametrically opposite from that of the continuum theories, though the objective-finding differential equations for fields, valid throughout a body - is exactly the same. It is perhaps fortuitous, or perhaps a sign that the equations actually represent some sort of physical "truth", that the forms of the equations resulting from the two approaches are so similar. For averaging theories, the region of space occupied by the material is thought of as being occupied by two different types of body, the suspended particles and the suspending medium. Each of the types of body is considered to be distinct in the sense that the join [11] of the bodies constitutes all of the space occupied by the composite body, while the meet is empty. Then each of the types of body is an ordinary continuum, and satisfies exactly the balance and constitutive laws expected of an ordinary continuum, that is,

$$
\begin{gathered}
\operatorname{div} \mathbf{v}_{a}=0 \\
\rho_{a} \grave{\mathbf{v}}_{a}=\rho_{a} \mathbf{b}_{a}+\operatorname{div} \mathbf{T}_{a}, \\
\left\{\mathbf{T}_{a}\right\}=g\left(\mathbf{v}_{b}, \text { and their derivatives }\right) .
\end{gathered}
$$

Here, of course, the bodies still are capable of momentum interaction, but unlike the previous situation, the micromechanical model for momentum interaction has a clear meaning. This is eight equations in eight unknowns, and thus is a determined system. Moreover, conditions for the difference of pressure such as Equation (6) now have a correct theoretical status, for now they are not field equations, rather, they are boundary conditions. Thus a determined system is obtained, and it is mathematically correct and physically plausible. The difficulty, of course, is that to formulate a boundary-value problem, a reasonable set of boundary and initial values for every particle in the system is needed. Such information normally is not available for any physical problem. Even if it were, finding a solution, with or without a computer as an intermediary, would be a nearly hopeless task. Moreover, even if such a solution were found, most of the information it contained would be of little use, because it would be too detailed. A plausible way to digest such data would be to average it in some sense. The usual approach in averaging theory is, not to go to the considerable trouble of averaging the solutions to (7), but rather to average (7) and then solve the averaged equations. Such an approach is highly appealing intellectually, but is fraught with mathematical difficulty. This paper is not the place to discuss such difficulties in detail. One, of course, is that the term "average" which has been used in a very vague

\footnotetext{
${ }^{5}$ Of course, the same argument can be made for the expressions in (4) for $\mathbf{m}_{a}$. There, however, the status of the equations is clear, because the appropriate micromechanical arguments can be used as motivation for the continuum theory, which then gives exact relations having the same status as field equations.
} 
way here, must be given a precise meaning. It is fortuitous that, for most of the averaging methods tried so far, the resulting equations have almost the form of the Equations (4) derived from the continuum theory. Unfortunately, for every method of averaging I have seen, though a determined system is averaged, the result of the averaging process is an underdetermined system, that is, the averaging process makes the closure problem reappear. Most readers will be familiar with why this happens without going through the details, for the averaging process always is similar to that used in turbulence theory and some of the extra terms are of the same form as Reynolds stresses. In a broad sense, then, though the continuum theory and the averaging theory start from different places and proceed by different methods, they end in approximately the same place: underdetermined systems of approximately the same form.

5. Sketch of a Theory for Dilute Suspensions. Previously in this paper, much has been made of the fact that most of the equations in the continuum theory have been "postulated". It is possible to interpret that terminology as meaning that they have been made up with no mathematical or physical basis. In fact, that is far from true. The kinetic theory of dilute monatomic gases for identical gas molecules is well-known and is commonly taught in courses for graduate students in science and engineering $[1,2,12]$. Much less well known is the fact that there is a similar theory for gases where there are a finite number of different types of molecules; in other words, a solution of several gases $[13,14]$. The resulting balance equations are exactly identical to those for the postulated theory of mixtures.

Here, I use the motivation of the kinetic theory of gases to support a mixture theory in an entirely different way. Most important for the discussion here is the fact that there is an exact definition of the pressure, and it is not the pressure "in" the particles, rather it is a momentum flux - an entirely different concept. ${ }^{6}$ Moreover, it is possible to force agreement of the theory with that of a viscous compressible gas, with the viscosity determined in terms of molecular parameters. I consider a dilute solution of particles in an inviscid fluid. Consider only the particles. They are an agglomeration of molecules, exactly like those in the theory of a monatomic gas, except that the scale of the molecules is somewhat larger than in a gas. Thus, precisely the same arguments can be used to motivate a continuum theory for the particle phase of the multiphase flow as is used for a gas. All of the expressions are the same, and e.g., one can accept the viscosity of the particle phase as a phenomenological coefficient, or one can consider it to be determined from molecular quantities, according to one's taste. In either case, unlike in the theories discussed in the previous two sections of this paper, it does have meaning. The equations

\footnotetext{
${ }^{6}$ In another paper in this volume, $O$. Walton uses the same definition in his computer molecular dynamics simulations.
} 
for the particle phase then are

$$
\begin{gathered}
\rho_{s}+\rho_{s} \operatorname{div} \mathbf{v}_{s}=0 \\
\rho_{s} \grave{\mathbf{v}}_{s}=\rho_{s} \mathbf{b}_{s}+\mathbf{m}+\operatorname{div} \mathbf{T}_{s}, \\
p_{s}=\hat{p}_{s}\left(\rho_{s}\right) \\
\mathbf{T}_{s}=\hat{\mathbf{T}}_{s}\left(\operatorname{sym} \operatorname{grad} \mathbf{v}_{s}\right) .
\end{gathered}
$$

This is a system of 5 equations in 5 unknowns, that is, a determined system. Now let the molecules be submerged in an incompressible fluid. Naturally, there will be an interaction between the particles and the fluid, and this interaction can be expressed as a constitutive equation for $\mathbf{m}$, which can be thought of as a part of the body force $\mathbf{b}_{f}$. Of course, the equations for the fluid phase are the expected ones,

$$
\begin{gathered}
\operatorname{div} \mathbf{v}_{f}=0 \\
\rho_{f} \grave{\mathbf{v}}_{f}=\rho_{f} \mathbf{b}_{f}-\mathbf{m}+\operatorname{div} \mathbf{T}_{f} \\
\mathbf{T}_{f}=\hat{\mathbf{T}}_{f}\left(\operatorname{sym} \operatorname{grad} \mathbf{v}_{f}\right) .
\end{gathered}
$$

again, a determined system. Thus, for a theory of this type, no closure problem exists. ${ }^{7}$

Generally in a theory of this type, one expects to see volume fractions appear intrinsically. Since the ideas here are for a very dilute, saturated suspension, the concept is not very important, except, perhaps, in the constitutive equation for $\mathbf{m}$ and in formulas for the "effective viscosity" $[5,12]$. Of course the idea can be introduced formally by setting

$$
\rho_{f}=\gamma_{f} \phi_{f}
$$

with $\gamma_{f}$ a constant, and

$$
\phi_{f}+\phi_{s}=1
$$

These substitutions introduce the same number of equations as unknowns.

Acknowledgments. Donald Drew, David McTigue, Mehrdad Massoudi, and Kathleen Pericak-Spector have been kind enough to discuss the ideas presented here with me. The work was supported by the United States Department of Energy. I express my deep appreciation to the Institute for Mathematics and its Applications for inviting me to lecture on this subject, and for help in preparation of the manuscript.

\footnotetext{
${ }^{7}$ In another paper in this volume, J. T. Jenkins and D.F. McTigue present a similar set of ideas for a very concentrated suspension. The kinetic theory is worked out in considerable detail. D. F. Mc Tigue also has presented a similar set of ideas in an unpublished lecture at the meeting of the American Geophysical Union held in December, 1988.
} 


\section{REFERENCES}

[1] C. Truesdell and R.G. Muncaster, Fundamentals of Maxwell's Kinetic Theory of a Simple Monotomic Gas, Academic Press, New York, 1980.

[2] W.G. Vincenti and C.H. Kruger, Jr., Introduction to Physical Gas Dynamics, Wiley, New York, 1965.

[3] Stephen L. Passman, Jace W. Nunziato and Edward K. Walsh, A Theory of Multiphase Mixtures, in Appendix $5 C$ of Rational Thermodynamics, 2nd edition, Springer-Verlag, New York, 1984.

[4] Ray M. Bowen, Theory of Mixtures, in Continuum Physics, Vol. III, edited by A.C. Eringen, Academic Press, New York, 1976.

[5] David F. McTigue, Richard C. Givler and Jace W. Nunziato, Rheological effects of nonuniform particle distributions in dilute suspensions, Journal of Rheology, 30, 5 (1986), pp. 1053-1076.

[6] Stephen L. Passman, Forces on the Solid Constituent in a Multiphase Flow, Journal of Rheology, 30, 5 (1986), pp. 1077-1083.

[7] J. D. Ramshaw and J. A. Trapp, Characteristics, stability, and short wavelength phenomena in two-phase flow equation systems, Nuclear Science and Engineering 66 (1978), pp. 93-102.

[8] C. PAUCHON AND S. BANNERJEE, Interphase moment um interaction effects in the averaged multifield model, Part I: Void propagation in bubbly flows, International Journal of Multiphase Flow, 12 (1986), pp. 555-573.

[9] C.J.S. Petrie, Elongational Flows, Pitman, London, 1979.

[10] C. Truesdell and W. Nold, The Non-Linear Field Theories of Mechanics, Handbuch der Physik $I I / 3$, Springer-Verlag, 1965. A modern discussion is given in P. Podio-Guidugli and M. Gurtin, The Thermodynamics of Constrained Materials, Archive for Rational Mechanics and Analysis, 51, (1973), pp. 192-208.

[11] C. Truesdell, A First Course in Rational Continuum Mechanics, Volume 1, Academic Press, New York, 1977.

[12] J.O. Hirschfelder, C. F. Curtiss And R. Byron Bird, Molecular Theory of Gases and Liquids, Wiley, 1954.

[13] J. C. Maxweld, On the Dynamical Theory of Gases, Philosophical Transactions of the Royal Society, London, 157 (1867), pp. 49-88.

[14] C. Truesdell, Sulle Basi di Termomeccanica, Accademia Nazionale dei Lincei, Rendiconti della Classe di Scienze Fisiche, Mathematiche e Naturali, Series 8, 22 (1957), pp. 33-38, 158-166; (An English translation by the author appears as On the Foundations of Mechanics and Energetics, Continuum Mechanics II, Gordon \& Breach, New York, 1965, 292-305. 